\title{
Moderate-Dose Treatment
}

National Cancer Institute

\section{Source}

National Cancer Institute. Moderate-Dose Treatment. NCI Thesaurus. Code C49342.

The regular strength or dosage quantity of a therapeutic agent prescribed to be taken at one time or at stated intervals. 